\title{
Primary cerebello-pontine angle melanoma: a case report
}

\author{
Arul Ponni ${ }^{1}$, Aniruddha Jagannatha ${ }^{2}$, Janaki Gururajachar ${ }^{1}$, Ritika Harjani ${ }^{1}$, Kirthi Koushik ${ }^{1}$, \\ Nirmala Subramanian ${ }^{2}$, Ramani Sowmya ${ }^{3}$, Ravi Varma ${ }^{2}$ \\ ${ }^{l}$ Department of Radiation Oncology, M.S. Ramaiah Medical College and Teaching Hospitals, Bangalore India. \\ ${ }^{2}$ Department of Neurosurgery, M.S. Ramaiah Medical College and Teaching Hospitals, Bangalore India. \\ ${ }^{3}$ Department of Ophthalmology, M.S. Ramaiah Medical College and Teaching Hospitals, Bangalore India.
}

Received May 06, 2014; Revised July 01, 2014; Accepted July 05, 2014; Published Online July 10, 2014

\section{Case Report}

\begin{abstract}
Intra-cranial melanomas are commonly metastatic from primary melanoma elsewhere in the body. The primary occurrence of a melanoma in the brain parenchyma is rare. We report a case of 38 year aged woman who presented with deviation of left eye and headache. On imaging, a space occupying lesion was found in the left Cerebello-pontine angle and a preoperative diagnosis of meningioma was made. She underwent left retro-mastoid sub-occipital craniectomy and excision of a black vascular tumor. Histopathological examination revealed a melanoma which was confirmed with Immunohistochemical assay. Search for dermal, mucous and ocular lesions were negative. She received adjuvant radiation to the post- operative tumor bed to 5400cGy in 30 fractions using Intensity Modulated Radiation Therapy technique along with concurrent Temozolomide. She tolerated the treatment well and is symptom free 12 months after treatment. Primary Melanomas are rare lesions of the Cerebello-pontine angle and its radiological features, evaluation and management have been discussed.
\end{abstract}

Keywords: Primary Central Nervous System Melanoma; Neurosurgery; Radiotherapy; Temozolomide

\section{Introduction}

Primary melanocytic lesions are rare tumors of Central Nervous System (CNS) with an incidence of 0.9 per 10 million. ${ }^{1,2}$ Though these tumors are seen anywhere along the leptomeningeal axis, they specifically cluster around the Brain stem and high cervical spinal cord because of high aggregation of melanocytes in the leptomeninges at these locations. ${ }^{3-5}$ The melanotic cells originate either from embryonic neuroectodermal cell rests or from the pial sheaths of the vasculature in the CNS. ${ }^{6}$ Focal tumors range from benign melanocytomas to malignant melanomas. Primary focal malignant melanomas are even rarer and account for $1 \%$ of all melanoma cases in the CNS. ${ }^{6}$ The diagnosis is essentially by exclusion of melanomas in the skin, mucosal membranes and eyes. ${ }^{4}$ Total excision, if achieved, results in longer sur-

Corresponding author: Arul Ponni; Department of Radiation Oncology, M.S. Ramaiah Medical College and Teaching Hospitals, Bangalore, India.

Cite this article as:

Ponni A, Jagannatha A, Gururajachar J, Harjani R, Koushik K, Subramanian N, Sowmya R, Varma R. Primary cerebello-pontine angle melanoma: a case report. Int J Cancer Ther Oncol 2014; 2(3):020315. DOI: 10.14319/ijcto.0203.15 vival. Adjuvant Radiation has a role in cases of incomplete or near-total excision. ${ }^{4}$ Temozolomide is an oral alkylating agent which crosses the Blood Brain Barrier (BBB) and is known to be effective in brain metastasis from malignant melanomas. ${ }^{7,8} \mathrm{We}$ reported a case of rare primary CNS malignant melanoma at Cerebello-pontine (CP) angle, managed successfully with gross total resection (GTR) and adjuvant radiotherapy along with concomitant Temozolomide. To the best of our knowledge this is the first case of primary CNS melanoma managed with concurrent Temozolomide.

\section{Case Report}

A thirty-eight-year woman presented to us with a history of medial deviation of the left eye (Figure 1) and double vision of two and a half years duration. She also complained of intermittent head ache of one year duration. Left eye showed hypertropia along with esotropia with IV and VI nerve palsy. Left pupil was mid-dilated, $5 \mathrm{~mm}$ in diameter and was reactive. Right pupil was normal. Corneal sensations were normal. Left Superior Oblique showed no intorsion on depression in adducted position. 


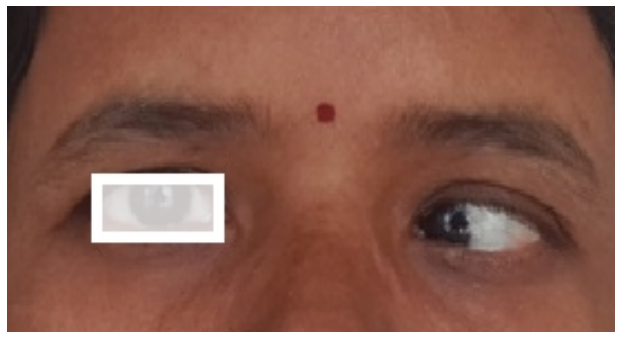

FIG. 1: Clinical photograph showing medial deviation of the left eye.

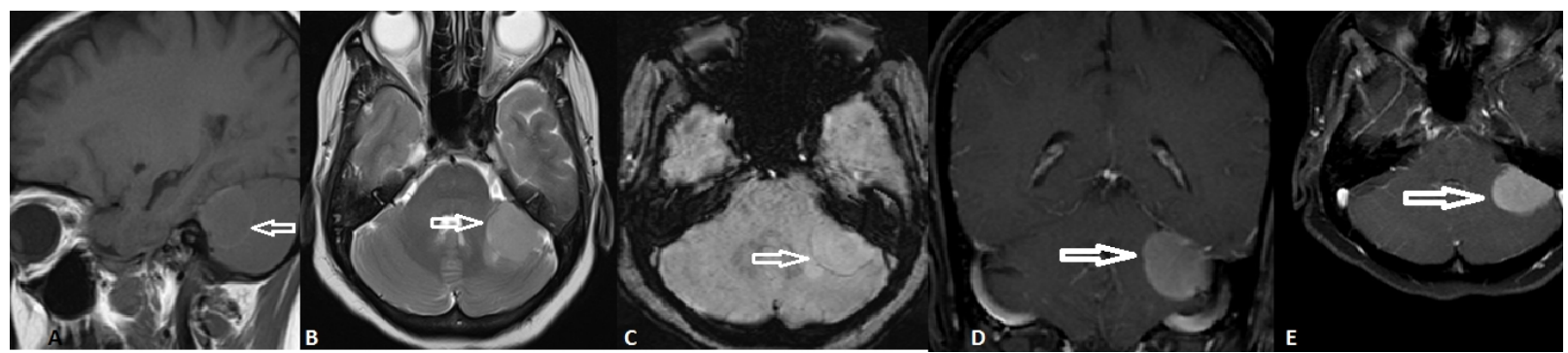

FIG. 2: Shows MRI brain with A) Sagittal T1W view with arrow showing a peripheral hyperintense rim on the isointense CP angle tumor; B) Axial T2W images; C) Susceptibility weighted images with peripheral rim; D) and E) contrast coronal and axial images with homogenous enhancement.

Plain and contrast magnetic resonance imaging (MRI) of the brain revealed an extra-axial, dural based lesion in the posterior part of the left $\mathrm{CP}$ angle adjacent to the petrous with a broad base. The lesion was compressing left Cerebellar Hemisphere. It was uniformly isointense on $\mathrm{T} 1 \mathrm{~W}$ images with a peripheral rim of hyper intensity, isointense on T2W images with a focal area of hyper intensity in the adjacent Cerebellum and showed homogeneous contrast enhancement. Peripheral rim of the lesion was well defined on susceptibility weighted image (SWI). VII-VIII nerve complex and the internal auditory canal were free from invasion. The rest of the brain was normal. With these features, a preoperative diagnosis of left CP angle Meningioma was made. (Figure 2A - E).

She underwent left retro mastoid sub-occipital craniectomy and gross total resection of the tumor. The tumor was jet black in colour (Figure 3), attached to the overlying meninges, soft and friable. Pial breach of the adjacent cerebellar folia was noted. Dural attachment of the tumor was coagulated after resection. Post-operative recovery was uneventful.

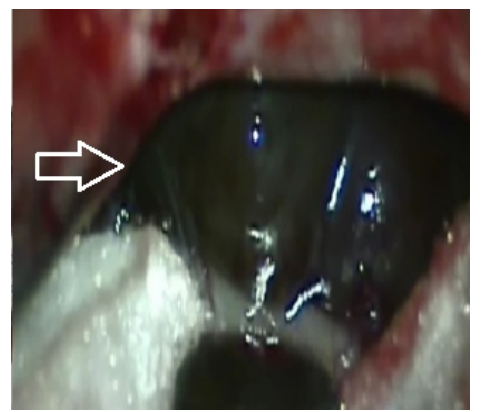

FIG. 3: Jet black tumor attached to the Dura.

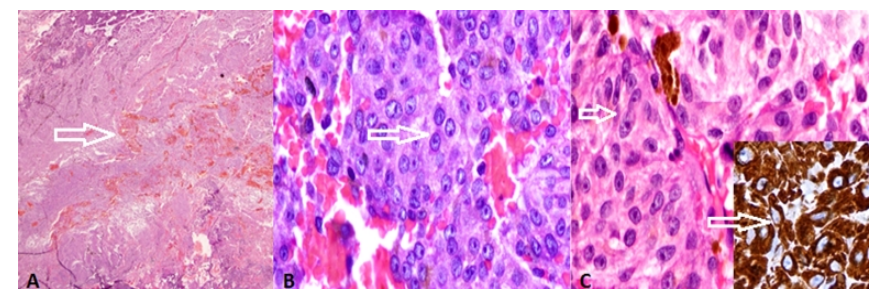

FIG. 4: A) Tumor with areas of Hemorrhages (arrow);B) Tumor cells with enlarged nuclei and single prominent nucleoli;C) Tumor with increased mitosis and HMB-45 positivity (inset).

Histopathological examination revealed a cellular tumor composed of relatively monotonous tumor cells in lobules separated by broad trabeculae. Areas of hemorrhage (Figure 4A) were noted in between. Tumor cells had enlarged round nuclei with single prominent nucleolus (Figure 4B). It was richly vascular and contained perivascular siderophages and melanophages. Focal areas within tumor showed fine melanin pigment in the cytoplasm. Extensive brain parenchymal infiltration by numerous tumor cell lobules was noted.

Immunohistochemistry was strongly positive for HMB-45 (Figure 4C) in all tumor cells suggesting melanocytic origin. S-100 and Vimentin were also positive. Possibility of a meningioma was ruled out as tumor was negative for an Epithelial Membrane Antigen (EMA). The MIB index of $8 \%$ at certain foci and $3-4 \%$ in other areas made us to consider this particular tumor a malignant one. The histopathological diagnosis was that of a CP angle malignant melanoma. Post-operative contrast MRI showed complete tumor excision and normal posterior fossa (Figure 5). 


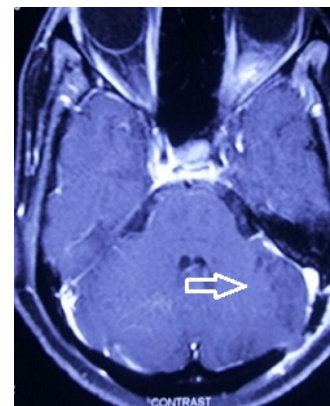

FIG. 5: Post op MRI showing complete excision.

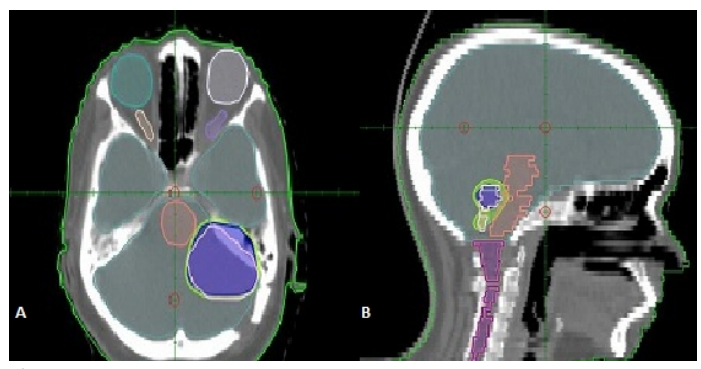

FIG. 6: A, B Radiotherapy planning CT images in axial and sagittal views delineating the target volume (pink line) and isodose distribution (blue line) conforming to the target volume avoiding dose to the adjacent brain stem and spinal cord.

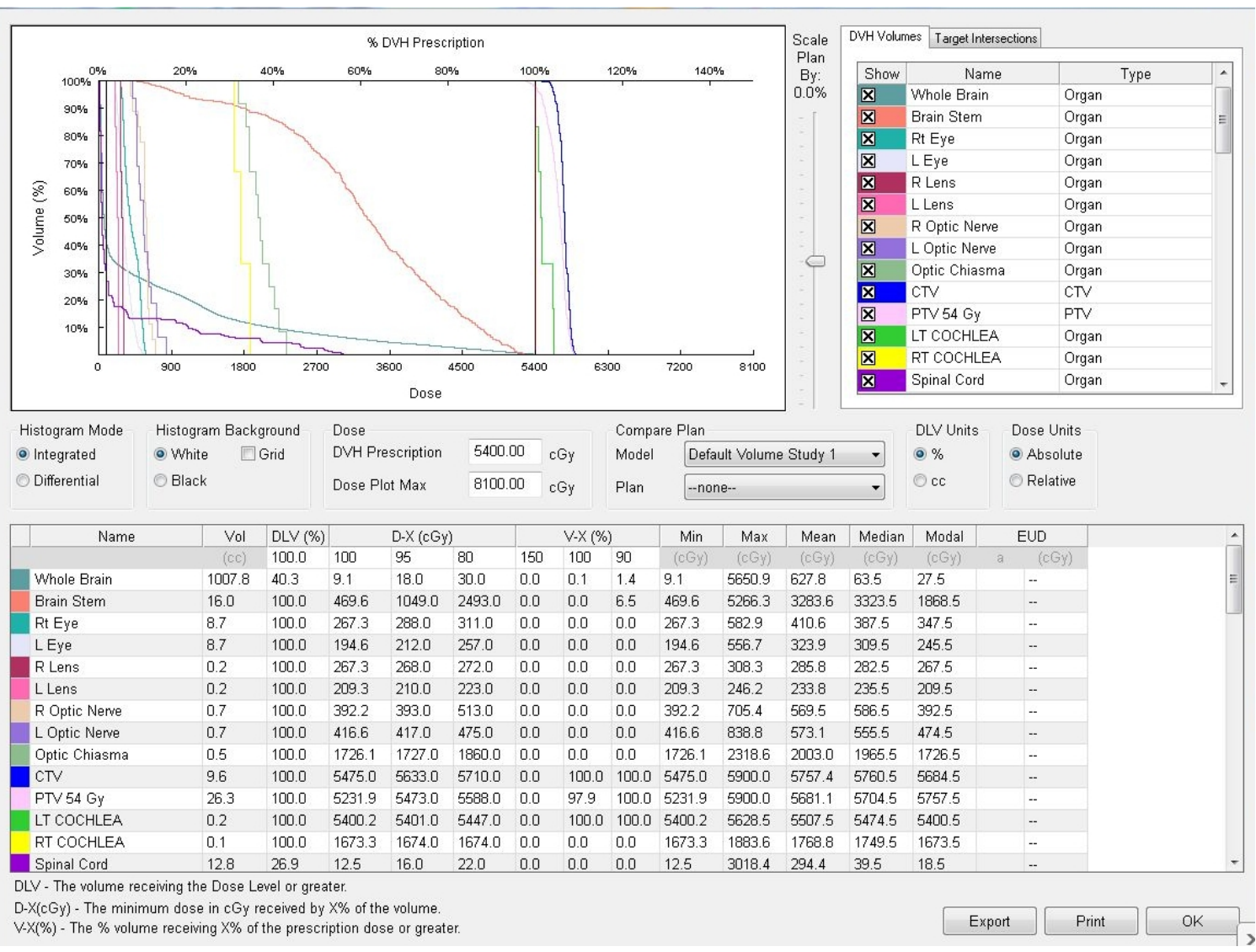

FIG. 7: Dose Volume Histogram showing Radiation dose to the surrounding vital structures within acceptable limits.

A detailed search for a primary melanoma was undertaken. Dermoscopy revealed no cutaneous lesions. Fundoscopy and B-scan in the eyes did not reveal any ocular lesions. ENT examination was normal. Postoperative Flourodeoxy Glucose-Positron Emission Computed Tomography ${ }^{18} \mathrm{~F}$ FDG-PET CT) did not show any abnormal uptake elsewhere in the body or in the primary site. The final diagnosis was a primary (nodular) malignant melanoma of Left CP angle with cerebellar infiltration.

She received adjuvant radiation in conventional fractionation to a total dose of $5400 \mathrm{cGy}$ in 30 fractions, 180cGy per fraction, five days per week to the tumor bed on 6MV Linear Accelerator using Intensity Modulated Radiation Therapy
(IMRT- Figure 6A, B) technique. She also received concurrent oral Temozolomide. The Dose Volume Histogram (DVH) showed adequate isodose coverage to the Planning Target Volume (PTV) while sparing the adjacent normal tissues (Figure 7). The daily dose of Temozolomide was $75 \mathrm{mg} / \mathrm{metre}$ square body surface area (BSA) to a dose of $100 \mathrm{mg}$ during Radiation for a period of six weeks. She tolerated the treatment well. Now at one year after diagnosis and surgery, she is asymptomatic, on a regular follow-up and is back to work with persistent (L) VI nerve palsy. One year follow-up MRI of the brain showed no recurrent mass (Figure 8). 


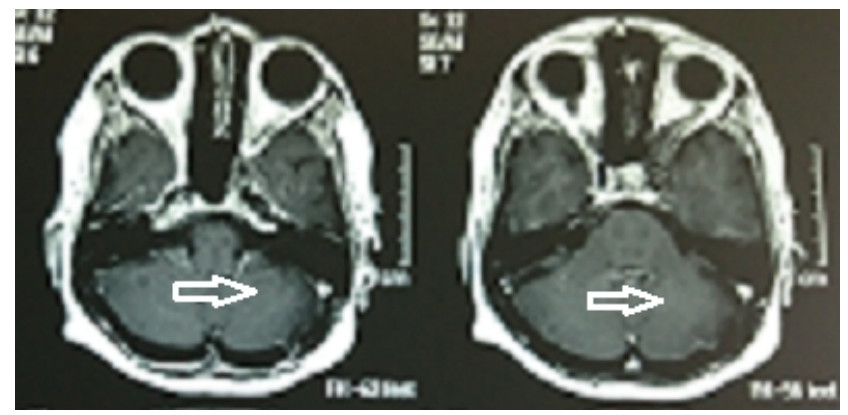

FIG. 8: One year follow-up MRI showing no tumor recurrence.

\section{Discussion}

Primary CNS melanocytic lesions can be broadly classified as diffuse leptomeningeal melanomatosis or focal melanocytic tumors. Diffuse lesions may arise de novo or can be a part of congenital neurocutaneous phakomatosis. Diffuse variety with leptomeningeal spread present with meningism, raised intracranial pressure and cranial nerve palsies. ${ }^{2,3}$ The clinical presentation of focal tumors depends on the location of the tumor.

Melanotic Schwannoma and Meningioma acquire melanin and they need to be considered in differential diagnosis of CP angle lesions with T2 shortening. Other pigmented lesions include Medulloblastoma, Choroid plexus papilloma and Astrocytoma. ${ }^{2}$ Phang et al., reviewed 24 cases of CP angle melanocytomas and noted that the mean age at presentation was 44.4 years, men were more frequently affected and symptoms persisted for an average of 5 months before diagnosis. MRI was typically T1 hyper and T2 hypo intense (melanotic). ${ }^{9}$ Melanomas are commonly hyper dense lesions (hemorrhage), with uniform or peripheral enhancement on CT scans. ${ }^{1}$ MRI of malignant melanoma of the CNS have two patterns viz, Melanotic and Amelanotic. Melanotic melanomas are typically hyper intense on $\mathrm{T} 1 \mathrm{~W}$ images and undergo T2 shortening to become hypo intense due to the paramagnetic effect of Melanin and blood products. Bleeding within the tumor can result in hetero-intense signals on MRI. Amelanotic melanomas are either iso or hypo on T1W images and iso to hyper intense on T2W scans. ${ }^{10}$ However Arriaga et al. noted that Amelanotic pattern on MRI was common at CP angle. ${ }^{10}$ Our patient had an amelanotic pattern, with atypical thin peripheral hyper intense rim on T1W scans which was well defined on SWI suggesting hemorrhage. ${ }^{3,10}$

Hayward in 1976 had defined primary CNS malignant melanomas based on clinical findings. According to him, solitary cerebral lesion, intramedullary or leptomeningeal involvement, hydrocephalus and pineal/pituitary tumors could be primary melanomas if no melanomatous lesion was found outside CNS. ${ }^{11} \mathrm{~A}$ meticulous search for a cutaneous, mucosal or ocular primary melanoma should prove unrevealing before a malignant melanoma can be accepted as indigenous to the CNS. In cases of CNS Melanomas, a workup for primary lesion picks an extra cranial tumor in approximately $90 \%$ of cases and $10 \%$ remain occult. ${ }^{3}$ Along with Cranial MRI, ${ }^{18} \mathrm{~F}$ FDG PET CT completes the search for primary. ${ }^{12,13}$ Whole body FDG PET CT is highly accurate (91\%) in diagnosing loco regional involvement and staging of melanoma. ${ }^{12}$

Based on MIB-1 staining, focal lesions can be further classified as benign melanocytomas $(0 \%-2 \%)$, intermediate grade melanocytic neoplasms (1\% - 4\%) and malignant melanomas (2\% - 15\%). ${ }^{14}$ Benign melanocytomas typically have variably pigmented melanocytic cells arranged in tight nests, sheets, or fascicles. In contrast, malignant melanomas have larger, cytologically atypical, pigmented tumor cells growing in loose nests or sheets, often with CNS invasion or necrosis. ${ }^{14}$ HMB-45 is an antimelanosomal antibody, which is specific for melanocytic tumors. It was found positive in $86 \%-97 \%$ of melanocytic tumors. S-100 is a sensitive marker for cells of Neural crest origin and lacks specificity. ${ }^{13,14}$ Both HMB-45 and S-100 were positive in our patient.

Prognosis in primary CNS malignant melanomas depends upon the location, extent of tumor excision, involvement of leptomeninges and MIB-1 labelling index. ${ }^{2}$ Diffuse leptomeningeal melanosis, though benign, carry dismal prognosis with a mean survival of 6.7 months. ${ }^{2}{ }^{2} 15$ Focal melanomas have a better prognosis with total excision. Overall survival upto 28 years have been reported after total resection of the tumor. In a study by Rodriguez et al, the mean survival of patients who underwent gross total removal, biopsy and subtotal removal, and no surgery was $19.58 \pm 2.3$ months, $9.30 \pm 2.4$ months, and $3.40 \pm 0.7$ months respectively. ${ }^{16}$

There are no treatment guidelines as these tumors are rare. Surgery remains the key modality of treatment. Subarachnoid spread of melanomas following surgery is known. ${ }^{2,3}$ Sometimes total excision becomes difficult in CP angle tumors due to brain infiltration. ${ }^{2}$ In a review, $80 \%$ of the cases with subtotal resection of CP angle melanocytomas died within 12 months of diagnosis. ${ }^{10}$ There are no effective adjuvant therapy guidelines. We treated our patient with post-operative radiation therapy as the literature suggests better recurrence free period. ${ }^{3}$ Wadasadawala et al. used adjuvant radiation up to $5400 \mathrm{cGy}$ in conventional fractionation. ${ }^{4}$

As the histopathology showed rich infiltration of the cerebellar foliae and high MIB-1 labelling index (8\%) suggesting high mitosis, this tumor was considered to be highly malignant and hence we considered adjuvant Radiation. Although Melanomas are known to be chemo-resistant, there are recent reports on the use of chemotherapeutic agents. Temozolomide and Dacarbazine (DTIC) have been used in malignant melanoma with brain metastasis., 7 Temozolomide was safe and with the ability to cross BBB, demonstrated activity in melanomatous brain metastasis. ${ }^{7}$ In a randomized 
trial comparing Temozolomide and Dacarbazine in patients with metastatic melanoma brain, the Temozolomide group showed comparable survival benefits with improved quality of life. ${ }^{7}$ A retrospective study done by Paul MJ et al. compared Temozolomide and DTIC in patients with advanced malignant melanoma. Results showed significant reduction in CNS relapse in the Temozolomide group compared to DTIC at 19 months follow-up. ${ }^{8}$ As Temozolomide has already been used in metastatic malignant melanoma of CNS with positive results and as the Primary CNS melanomas have similar cell lineage, Temozolomide was chosen as the chemotherapeutic agent. ${ }^{7,8}$ The patient tolerated the therapy satisfactorily. The role of concurrent Temozolomide in the adjuvant setting needs further evaluation both in primary and metastatic CNS melanomas. She is planned for a close long term follow up as it is still early in the disease.

\section{Conclusion}

Primary CNS melanoma is difficult to diagnose preoperatively. Typically the MRI picture, gross features of the tumor, MIB-1 proliferative index, immunohistochemistry and ${ }^{18}$ F-FDG PET CT plays a major role in the diagnosis of primary CNS malignant melanoma. Radical excision is the main modality of treatment. Concurrent chemoradiation in adjuvant setting for primary CNS malignant melanoma is beneficial.

\section{Conflict of interest}

The authors declare that they have no conflicts of interest. The authors alone are responsible for the content and writing of the paper.

\section{Acknowledgement}

We thank Dr. Yasha TC, professor and head, department of neuropathology, National institute of mental health and neurosciences (NIMHANS), Bangalore.

\section{References}

1. Kan P, Shelton C, Townsend J, Jensen R. Primary Malignant Cerebellopontine Angle Melanoma Presenting as a Presumed Meningioma: Case Report and Review of the Literature. Skull Base 2003; 13:159-66.

2. Bhandari L, Alapatt J, Govindan A, Sreekumar T.Primary cerebellopontine angle melanoma: a case report and review. Turk Neurosurg 2012; 22:469-74.
3. Shah I, Imran M, Akram R, et al. Primary intracranial malignant melanoma. J Coll Physicians Surg Pak 2013; 23:157-9.

4. Wadasadawala T, Trivedi S, Gupta T, et al. The diagnostic dilemma of primary central nervous system melanoma. J Clin Neurosci 2010; 17:1014-7.

5. Larson TC 3rd, Houser OW, Onofrio BM, Piepgras DG. Primary spinal melanoma. J Neurosurg 1987; 66:47-9.

6. Farrokh D, Fransen P, Faverly D. MR findings of a primary intramedullary malignant melanoma: case report and literature review. AJNR Am J Neuroradiol 2001; 22:1864-6.

7. Agarwala SS, Kirkwood JM, Gore M, et al. Temozolomide for the treatment of brain metastases associated with metastatic melanoma: a phase II study. J Clin Oncol 2004; 22:2101-7.

8. Paul MJ, Summers Y, Calvert AH, et al. Effect of temozolomide on central nervous system relapse in patients with advanced melanoma. Melanoma Res 2002; 12:175-8.

9. Phang I, Elashaal R, Ironside J, Eljamel S. Primary cerebellopontine angle melanocytoma: review. $J$ NeurolSurg Rep 2012; 73:25-31.

10. Escott EJ. A variety of appearances of malignant melanoma in the head: a review. Radiographics 2001; 21:625-39.

11. Hayward RD. Malignant melanoma and the central nervous system. A guide for classification based on the clinical findings. J Neurol Neurosurg Psychiatry 1976; 39:526-30.

12. Fuster D, Chiang S, Johnson G, et al. Is 18F-FDG PET more accurate than standard diagnostic procedures in the detection of suspected recurrent melanoma? J Nucl Med 2004; 45:1323-7.

13. Belhocine TZ, Scott AM, Even-Sapir E, et al. Role of nuclear medicine in the management of cutaneous malignant melanoma. J Nucl Med 2006; 47:957-67.

14. Brat DJ, Giannini C, Scheithauer BW, et al. Primary melanocytic neoplasms of the central nervous systems. Am J SurgPathol 1999; 23:745-54.

15. Dechaphunkul A, Kayasut K, Oearsakul T, et al. Common presentation in an uncommon disease: case report of a patient with primary diffuse leptomeningealmelanocytosis. J Clin Oncol 2011; 29:e816-8.

16. Rodriguez y Baena R, Gaetani P, Danova M, et al. Primary solitary intracranial melanoma: case report and review of the literature. Surg Neurol 1992; 38:26-37. 